\author{
nature \\ structural \& \\ molecular biology
}

\title{
Real-time assembly landscape of bacterial 30 S translation initiation complex
}

\author{
Pohl Milón ${ }^{1}$, Cristina Maracci ${ }^{2}$, Liudmila Filonava $^{1}$, Claudio O Gualerzi ${ }^{2}$ \& Marina V Rodnina ${ }^{1}$ \\ Initiation factors guide the ribosome in the selection of mRNA and translational reading frame. We determined the kinetically \\ favored assembly pathway of the $30 \mathrm{~S}$ preinitiation complex (30S PIC), an early intermediate in $30 \mathrm{~S}$ initiation complex formation \\ in Escherichia coli. IF3 and IF2 are the first factors to arrive, forming an unstable 30S-IF2-IF3 complex. Subsequently, IF1 joins \\ and locks the factors in a kinetically stable 30S PIC to which fMet-tRNA ${ }^{\text {fMet }}$ is recruited. Binding of mRNA is independent of \\ initiation factors and can take place at any time during 30S PIC assembly, depending on the cellular concentration of the mRNA \\ and the structural determinants at the ribosome-binding site. The kinetic analysis shows both specific and cumulative effects of \\ initiation factors as well as kinetic checkpoints of mRNA selection at the entry into translation.
}

During initiation of protein synthesis, the ribosome recruits an mRNA for translation and establishes the reading frame by binding initiator tRNA to the start codon in the P site of the small (30S) ribosomal subunit. In bacteria, initiation requires three initiation factors, IF1-IF3, and proceeds in several steps ${ }^{1-3}$. In the first step, mRNA, initiation factors and initiator fMet-tRNA ${ }^{\text {fMet }}$ assemble on the 30 S subunit to form a transient 30S PIC (Fig. 1a). The ribosome binds to single-stranded regions of the mRNA, exploiting the base complementarity between the Shine-Dalgarno sequence (when present) of the mRNA and the anti-Shine-Dalgarno sequence in $16 \mathrm{~S}$ rRNA as a guide. fMet-tRNA ${ }^{\mathrm{fMet}}$ is recruited through IF2, which is already bound to the 30 S subunit ${ }^{4}$. The recognition of the start codon by fMet-tRNA ${ }^{\text {fMet }}$ triggers conformational changes that convert the 30S PIC to a functionally competent 30 S initiation complex (30S IC; Fig. 1b) ${ }^{3}$. Finally, the 30 S IC is joined by the large (50S) ribosomal subunit, forming the 70S IC, which is poised for translation of the selected $\mathrm{mRNA}^{5-9}$. The assembly of the $30 \mathrm{~S}$ PIC, its rearrangement to the 30 S IC and the transition to the 70S IC are important checkpoints that control the frequency at which a particular mRNA is translated and prevent out-of-frame initiation, which would lead to synthesis of aberrant polypeptides ${ }^{5-7,10}$.

Late events in translation initiation, particularly the transition from the 30 S IC to the 70 S IC, have been investigated in detail ${ }^{5-9}$. In contrast, the timing of events and the compositional dynamics of the 30S PIC assembly are unknown. Binding of mRNA to the $30 \mathrm{~S}$ subunit requires a single-stranded mRNA region in the vicinity of the translation initiation region but is independent of 30S PIC composition ${ }^{10,11}$. Three initiation factors, mRNA and initiator tRNA bind to the $30 \mathrm{~S}$ subunit independently of each other, suggesting parallel pathways of 30S PIC assembly ${ }^{1}$. However, it is unknown whether there is a preferred pathway and whether the kinetically favored order of initiation factor binding controls mRNA and start-codon selection.
The initial docking of the factors may be followed by conformational rearrangements that lead to maturation of the complex, as has been suggested for IF2 (refs. 4,12) and mRNA ${ }^{10,13}$.

In this study we tracked the dynamics of 30 S PIC formation in real time, monitoring transient changes of fluorescence resonance energy transfer (FRET) between ligands in the 30S PIC. We asked the following questions: what are the timing and choreography of $30 \mathrm{~S}$ PIC assembly, and how do the factors exert control over each other? On the basis of the full set of kinetic constants, we identify potential checkpoints for mRNA selection and provide a time axis for structural studies on translation initiation.

\section{RESULTS}

We determined kinetic parameters of interactions between 30S subunits and different combinations of ligands by the stopped-flow technique (Online Methods). Fluorescent labels were introduced into each of the initiation factors, fMet-tRNA ${ }^{\text {fMet }}$ and mRNA (Supplementary Table 1$)^{14}$. Fluorescence and FRET changes due to ligand binding to the $30 \mathrm{~S}$ subunit were followed in real time. Individual rate constants $(k)$ are labeled with subscripts comprising several digits. The first digit indicates the factor under study, and the following digits indicate the factor(s) prebound to the $30 \mathrm{~S}$ subunit; for example, $k_{312}$ is the association rate constant of IF3 binding to a 30S-IF1-IF2 complex. Dissociation rate constants are labeled analogously with a minus sign. In the experiments carried out with the $30 \mathrm{~S}$ IC, the respective rate constants are labeled $k_{\text {IC }}$ and $k_{-\mathrm{IC}}$. For two-step binding mechanisms, the first and second steps are additionally indicated by 'a' and 'b', respectively.

\section{Recruitment and locking of IF1 in 30S PIC and 30S IC}

IF1 $(8.2 \mathrm{kDa})$ is a compact globular protein (Fig. 1b) without cysteines. We introduced a cysteine by replacing the nonconserved Asp4, and

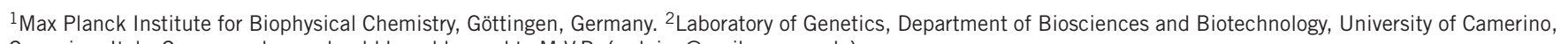
Camerino, Italy. Correspondence should be addressed to M.V.R. (rodnina@mpibpc.mpg.de). 
a

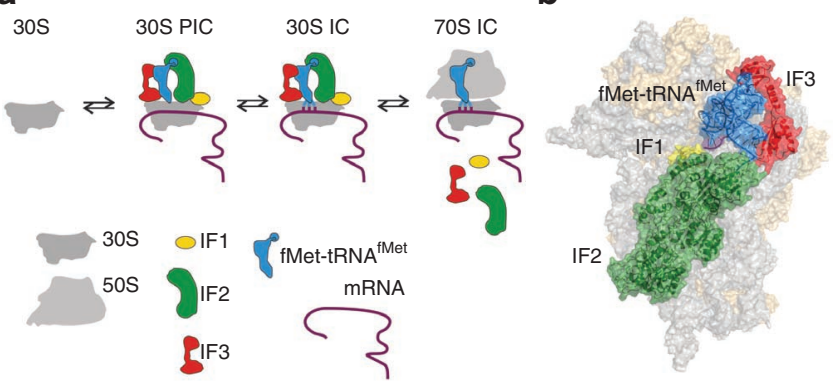

Figure 1 Translation initiation in bacteria. (a) Schematics of initiation. For explanations, see text. (b) Structural model of the 30S IC based on cryo-EM reconstruction ${ }^{15}$ with IF1 (yellow), IF2 (green), IF3 (red) and fMet-tRNA ${ }^{\text {fMet }}$ (blue).

labeled the protein with Alexa 555-maleimide (Invitrogen, Germany), yielding fluorescent, fully active $\mathrm{IF}_{4}(\mathrm{Alx} 555)$ (Supplementary Fig. 1 and ref. 7). We used $\mathrm{IF}_{4}(\mathrm{Alx} 555)$ as FRET acceptor for Alx488modified IF2 or IF3, or for fluorescein-labeled fMet-tRNA ${ }^{\text {fMet }}$ (Supplementary Table 1). Binding of IF1 to the 30S subunit led to single-exponential time courses (Fig. 2a). The $k_{\text {app }}$ values increased linearly with IF1 concentration, consistent with a one-step binding mechanism (Fig. 2b, inset). Association rate constants were in the range of 7-20 $\mu \mathrm{M}^{-1} \mathrm{~s}^{-1}$ (Fig. $2 \mathbf{b}$ and Table 1). IF1 binding to the $30 \mathrm{~S}$ subunit was somewhat faster without IF2 than with it; this may be explained by partial shielding of the IF1-binding site by the NTD of IF2 (Fig. 1b $)^{15}$. The 30S-IF1-IF2 or 30S-IF1-IF3 complexes dissociated readily, with dissociation rate constants of $2 \mathrm{~s}^{-1}$ and $0.7 \mathrm{~s}^{-1}$, respectively (Fig. 2c and Supplementary Fig. 2a). The presence of IF2 and IF3 together had a cumulative effect, leading to a kinetically locked complex $\left(k_{-123}=0.02 \mathrm{~s}^{-1}\right)$, with a $K_{\mathrm{d}}$ of $\sim 2 \mathrm{nM}$. Addition of mRNA did not change the kinetics of IF1 binding (Supplementary Fig. 3). The addition of $\mathrm{fMet}^{-\mathrm{tRNA}}{ }^{\mathrm{fMet}}$ together with mRNA containing an AUG codon led to a further ten-fold stabilization of IF1 in the complex (Fig. 2c and Table 1). Notably, the release of IF1 from the 30S IC was 70-fold faster without IF3 than with it $\left(0.0025 \mathrm{~s}^{-1}\right.$ versus $\left.0.17 \mathrm{~s}^{-1}\right)$. We could not monitor binding of IF1 to vacant 30 S subunits (without FRET donor or acceptor) because we observed no fluorescence intensity change in IF1; the $K_{\mathrm{d}}$ value of the $30 \mathrm{~S}-\mathrm{IF} 1$ complex, as estimated from the thermodynamic cycle that includes IF3 binding, was $0.9 \mu \mathrm{M}$, consistent with values measured earlier by different methods $(0.1-2 \mu \mathrm{M})^{16,17}$.

Recruitment and conformational rearrangements of IF2

IF2 (97 kDa; Fig. 1b) is a large GTPase that comprises an N-terminal domain (NTD), a GTP-binding domain and a C-terminal domain
(CTD). Escherichia coli IF2 has three intrinsic cysteines, Cys599 in the GTP-binding domain and two cysteines in the CTD. Labeling with Atto465- or Alx488-maleimides leads to the preferential modification of Cys599, whereas the other two cysteine residues, which are buried inside the structure ${ }^{18}$, are labeled to a very small extent $(<10 \%)$, as estimated from the labeling efficiency of the IF2 mutant C599S.

IF2 ${ }_{599}$ (Atto465) was used as FRET donor for the acceptor fluorophore, Alx555, placed into IF1 and IF3 (Fig. 3a). When only IF3 was present on the 30 S subunit, single-exponential time courses of IF2 binding were obtained. The rate constants calculated from the concentration dependence (Fig. 3b) were $280 \mu \mathrm{M}^{-1} \mathrm{~s}^{-1}$ (association) and $30 \mathrm{~s}^{-1}$ (dissociation). The chase experiment yielded a similar value for the dissociation rate constant. The resulting $K_{\mathrm{d}}$ of the complex was $50-110 \mathrm{nM}$, similar to the value obtained for the $30 \mathrm{~S}-\mathrm{IF} 2$ complex without IF3 $\left(K_{\mathrm{d}}=40-60 \mathrm{nM}\right)^{19}$. In the presence of IF3 together with IF1 or with 30 S IC, time courses of IF2 binding were double-exponential. The $k_{\text {app } 1}$ values increased linearly with concentration, whereas $k_{\text {app2 }}$ was concentration independent (Fig. 3b), indicating a two-step binding mechanism (Fig. 3c). The forward rate constants of IF2 binding and rearrangement were similar for 30S-IF1-IF3 and 30S IC: $k_{2 \mathrm{a}}=220-320 \mu \mathrm{M}^{-1} \mathrm{~s}^{-1}$ and $k_{2 \mathrm{~b}}=2-6 \mathrm{~s}^{-1}$ (Table 1). In contrast, the dissociation of IF2 from the two complexes was markedly different; $k_{-213 \mathrm{~b}}=1 \mathrm{~s}^{-1}$ for the 30S-IF1-IF2-IF3 complex (Fig. 3d and Supplementary Fig. 2b) compared with $k_{-2 \mathrm{ICb}}=0.015 \mathrm{~s}^{-1}$ from the 30S IC (Table 1). We could not determine the complete sets of rate constants for IF2 binding to the vacant 30 S subunit or 30S-IF1 complex owing to the lack of a suitable signal change; however, we made some comparisons to reported values ${ }^{12}$. The two-step mechanism and the values of rate constants reported here agree well with the data on IF2 recruitment to the 30S-IF1-IF3 complex obtained by monitoring a fluorophore in IF2 (ref. 12). Reported $k_{2}$ values for IF2 recruitment are $100-300 \mu \mathrm{M}^{-1} \mathrm{~s}^{-1}$ for different combinations of IF3, fMet-tRNA ${ }^{\mathrm{fMet}}$ and the mRNA ${ }^{12}$, suggesting that IF2 binds rapidly to any 30 S complex. Chase experiments (Fig. 3d and Supplementary Table 2) showed that (i) the addition of mRNA did not affect IF2 dissociation from the $30 \mathrm{~S}$ subunit; (ii) dissociation of IF2 from the 30S-IF3 complex was ten-fold slower with IF1 than without it; (iii) IF2 release was not influenced by IF3 and (iv) the presence of fMet-tRNA ${ }^{\text {fMet }}$ stabilized the binding of IF2, particularly in the presence of IF1.

\section{IF3 recruitment during initiation and ribosome recycling}

IF3 $(20 \mathrm{kDa})$ comprises two domains (Fig. 1b). The NTD contains an intrinsic Cys65, which we replaced with serine. Cysteine was introduced at position 166 in the CTD, which carries out most functions of IF3 (ref. 20). We labeled Cys166 with Alx488-maleimide (IF3 ${ }_{166}(\mathrm{Alx} 488)$ ), a FRET donor to the nonfluorescent acceptor Atto540Q, which was placed at the $5^{\prime}$ end of the mRNA.
Figure 2 Recruitment of IF1 to the 30S PIC (a) Time courses of IF $1_{4}(\mathrm{Al} \times 555)$ binding to the 30 S subunit with IF2 ${ }_{757}($ Alx488) alone, IF3 ${ }_{166}$ (Alx488) alone, IF2 and IF3 ${ }_{166}$ (Alx488) together, and IF2, IF3 166 (AIx488), mRNA and fMet-tRNA ${ }^{\text {fMet }}(30 \mathrm{~S} I \mathrm{C})$ as monitored by FRET changes. (b) Concentration dependence of $k_{\text {app }}$ values of IF1 binding to 30 S subunits with IF2

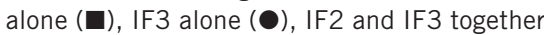
$(\mathbf{\Delta})$ and $30 \mathrm{~S} \mathrm{IC}(\boldsymbol{\nabla})$. Components were mixed in these combinations, with final concentrations after mixing in parentheses: 30 S subunits $(0.05 \mu \mathrm{M})$, IF3 $_{166}$ (Alx488) $(0.06 \mu \mathrm{M})$, IF2 $(0.15 \mu \mathrm{M})$ or IF2 757 (Alx488) $(0.11 \mu \mathrm{M})$ with GTP $(0.25 \mathrm{mM})$, fMet-tRNA ${ }^{\text {fMet }}(0.15 \mu \mathrm{M})$ and mRNA $(0.15 \mu \mathrm{M})$. Values are mean \pm s.d. from eight to ten time courses. Inset, kinetic scheme of binding. (c) Summary of $k_{-1}$ values for IF1 release measured by chase (see Table 1 ; for 30 S IC without IF3, $k_{-1}=0.17 \mathrm{~s}^{-1}$ ). Representative time courses of respective reactions are in Supplementary Figure 2. Values are mean and s.d. from eight to ten time courses.
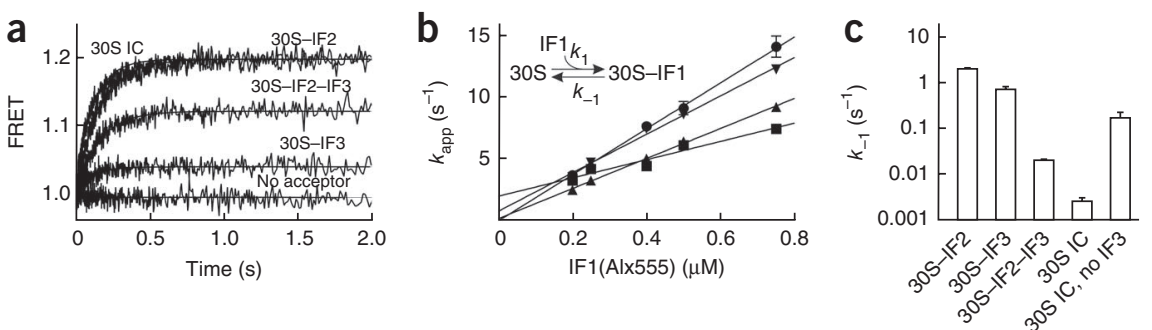
Table 1 Summary of association and dissociation rate constants of initiation factor binding during $30 \mathrm{~S}$ PIC assembly

\begin{tabular}{lccc}
\hline IF1 & & & \\
complex & $k_{1}\left(\mu \mathrm{M}^{-1} \mathrm{~s}^{-1}\right)^{\mathrm{a}}$ & $k_{-1}\left(\mathrm{~s}^{-1}\right)^{\mathrm{b}}$ & $K_{\mathrm{d}}(\mathrm{nM})^{\mathrm{c}}$ \\
\hline 30S-IF2 & $7 \pm 1$ & $2 \pm 0.1$ & $290 \pm 40$ \\
30S-IF3 & $20 \pm 1$ & $0.7 \pm 0.1$ & $35 \pm 5$ \\
30S-IF3-IF2 & $12 \pm 1$ & $0.02 \pm 0.001$ & $1.7 \pm 0.2$ \\
30S IC & $16 \pm 1$ & $0.0025 \pm 0.001$ & $<1$ \\
\hline
\end{tabular}

\begin{tabular}{lccccc}
\hline IF2 & & & & \\
complex & $k_{2 a}\left(\mu M^{-1} s^{-1}\right)^{a}$ & $k_{-2 a}\left(s^{-1}\right)$ & $k_{2 b}\left(s^{-1}\right)^{d}$ & $k_{-2 b}\left(s^{-1}\right)$ & $K_{d}(n M)$ \\
\hline 30S-IF3 & $280 \pm 60$ & $12 \pm 0.6^{b}$ & - & - & $43 \pm 10$ \\
30S-IF3-IF1 & $220 \pm 15$ & $15 \pm 3$ & $2 \pm 0.2$ & $1 \pm 0.1$ & $35 \pm 10$ \\
30S IC & $320 \pm 50$ & $\sim 0$ & $6 \pm 2$ & $0.015 \pm 0.002^{b}$ & ND \\
\hline
\end{tabular}

\begin{tabular}{lccccc} 
30S IC & $320 \pm 50$ & $\sim 0$ & $6 \pm 2$ & $0.015 \pm 0.002^{\mathrm{b}}$ & ND \\
\hline IF3 & & & & \\
complex & $\mathrm{K}_{3 \mathrm{a}}\left(\mu \mathrm{M}^{-1} \mathrm{~s}^{-1}\right)$ & $\mathrm{k}_{-3 \mathrm{a}}\left(\mathrm{s}^{-1}\right)$ & $\mathrm{k}_{3 \mathrm{~b}}\left(\mathrm{~s}^{-1}\right)$ & $\mathrm{k}_{-3 \mathrm{~b}}\left(\mathrm{~s}^{-1}\right)$ & $\mathrm{K}_{\mathrm{d}}(\mathrm{nM}) \mathrm{g}$ \\
\hline 30S & $1,160 \pm 20$ & $36 \pm 2$ & $55 \pm 1$ & $0.8 \pm 0.1$ & $3 \pm 0.2$ \\
30S-IF1 & $1,100 \pm 50$ & $9 \pm 2$ & $37 \pm 3$ & $0.08 \pm 0.02$ & $<0.1$ \\
30S-IF2 & $1,040 \pm 20$ & $34 \pm 3$ & $51 \pm 1$ & $0.7 \pm 0.06$ & $0.2 \pm 0.05$ \\
30S-IF1-IF2 & $960 \pm 20$ & $16 \pm 1$ & $34 \pm 1$ & $0.02 \pm 0.001$ & $<0.1$ \\
30S IC & $1,070 \pm 50$ & $10 \pm 2$ & $11 \pm 2$ & $3 \pm 0.5$ & $2.5 \pm 0.4$ \\
30S-tRNA & $670 \pm 10$ & $4 \pm 1$ & $0.9 \pm 0.1$ & $\sim 0$ & ND
\end{tabular}

${ }^{a}$ Association rate constants determined from slope and $y$-axis intercept of linear concentration dependence of $k_{\text {app }}$ (for a one-step mechanism) or $k_{\text {app } 1}$ (for a two-step mechanism); values are mean $\pm \mathrm{s}$.d. of linear fitting. ${ }^{b}$ Dissociation rate constant determined in chase experiments (Supplementary Fig. 2). ${ }^{c} K_{d}$ calculated from $k_{-1}: k_{1}$. ${ }^{d}$ See scheme in Figure $3 c$. The $k_{2 b}$ value was calculated from the hyperbolic fit of the $k_{a p p 2}$ concentration dependence and the $k_{-2 b}$ value was determined by chase; $k_{2 \mathrm{~b}}=k_{\mathrm{app} 2}-k_{-2 \mathrm{~b}}$, where $k_{-2 \mathrm{~b}}=k_{\mathrm{off}}{ }^{\mathrm{e}} K_{\mathrm{d}}$ was calculated from $k_{-2}: k_{2}$ or $\left(k_{-2 \mathrm{a}} \times k_{-2 \mathrm{~b}}\right) /\left(k_{2 \mathrm{a}} \times k_{2 \mathrm{~b}}\right) .{ }^{\mathrm{f}}$ All constants were obtained by global fitting of time courses (Online Methods); values are mean \pm s.d. of global fit. ${ }^{g} K_{d}$ was calculated from two consecutive steps $\left(k_{-3 a} \times k_{-3 b}\right) /\left(k_{3 a} \times k_{3 b}\right) . N D$, not determined.

Upon binding of $\operatorname{IF}_{166}(\mathrm{Alx} 488)$ to 30 S subunits either with mRNA alone or carrying any combination of factors, the fluorescence of Alx488 decreased owing to FRET quenching by 30S-bound mRNA(AttoQ). The FRET change was biphasic (Fig. 4a), and the concentration dependence of $k_{\text {app } 1}$ and $k_{\text {app2 }}$ (Supplementary Fig. 4) was consistent with a two-step binding mechanism (Fig. 4b). We first estimated values of the rate constants from linear and hyperbolic fitting of $k_{\text {app } 1}$ and $k_{\text {app2 }}$, respectively, and further refined them by global fitting of the combined time courses (Fig. 4c). IF3 associated with the $30 \mathrm{~S}$ subunit extremely rapidly $\left(k_{3 \mathrm{a}} \approx 1,000 \mu \mathrm{M}^{-1} \mathrm{~s}^{-1}\right)$, regardless of the presence of the other two factors. The dissociation of IF3 from the initial complex was somewhat faster without IF1 $\left(k_{-3 \mathrm{a}}\right.$ and $k_{-32 \mathrm{a}}$ of $\left.\sim 35 \mathrm{~s}^{-1}\right)$ than with it $\left(k_{-31 \mathrm{a}}=9 \mathrm{~s}^{-1}\right.$ and $\left.k_{-312 \mathrm{a}}=16 \mathrm{~s}^{-1}\right)$. The early complex rearranged rapidly $\left(34-55 \mathrm{~s}^{-1}\right)$ into a complex in which IF3 was stabilized; the extent of stabilization depended on IF1. Without IF1, the rearrangement was readily reversible $\left(k_{-3 \mathrm{~b}}\right.$ and $k_{-32 \mathrm{~b}}$ of $\left.0.7-0.8 \mathrm{~s}^{-1}\right)$, whereas with IF1, IF3 dissociated very slowly $\left(k_{-31 \mathrm{~b}}\right.$ and $k_{-312 \mathrm{~b}}$ of $0.02-0.08 \mathrm{~s}^{-1}$; Table 1). Addition of fMet-tRNA ${ }^{\text {fMet }}$ and mRNA to

Figure 3 Recruitment of IF2 to the 30S PIC. (a) Time courses of IF2 599 (Atto465) binding to 30 S subunit with IF3 ${ }_{166}$ (Alx555) alone, IF1 and IF $3_{166}$ (AIx555) together, or $30 \mathrm{SIC}$ as monitored by FRET changes. (b) Concentration dependence of $k_{\text {app }}$ values of IF2 binding to 30-IF3 $(\boldsymbol{\nabla})$, 30S-IF1-IF3 ( $\left.k_{\text {app } 1}, \mathbf{\square} ; k_{\text {app } 2}, \square\right)$ or 30 IC $\left(k_{\text {app } 1}, \mathbf{0} ; k_{\text {app } 2}, 0\right) .30 S$ subunits (increasing concentrations) were mixed with IF2 599 (Atto465) $(0.02 \mu \mathrm{M})$, GTP $(0.25 \mathrm{mM})$, and IF3 ${ }_{166}($ Alx555) $(0.45 \mu \mathrm{M})$, with or without IF1 $(1 \mu \mathrm{M})$, with or without fMet-tRNA ${ }^{\mathrm{fMet}}(0.45 \mu \mathrm{M})$ and mRNA (m002, $0.45 \mu \mathrm{M}$ ); the respective complexes are collectively

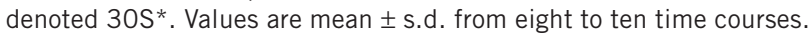
(c) Schematic of IF2 binding followed by a rearrangement step. (d) Summary of $k_{\text {off }}$ values of IF2 from various $30 \mathrm{~S}$ complexes. Time courses of chase experiments and $k_{\text {off }}$ values are summarized in Supplementary Figure 2 and Supplementary Table 2. Values are mean and s.d. from eight to ten time courses. the 30S-IF1-IF2-IF3 complex (that is, the formation of the 30S IC) led to similar rate constants for the first step but a 150 -fold greater dissociation rate of IF3 (to $k_{-\mathrm{IC}}=3 \mathrm{~s}^{-1}$; Table 1 and Fig. 4d).

In addition to its role in initiation, IF3 acts at the ribosome recycling step ${ }^{21,22}$. IF3 is recruited to the $30 \mathrm{~S}$ subunit after disassembly of the post-termination ribosome with the help of the ribosome recycling factor (RRF) and elongation factor G (EF-G) $)^{21,22}$, precluding reassociation of the $50 \mathrm{~S}$ subunit. To study the timing of IF3 recruitment to the ribosome during recycling, we prepared post-termination complexes with mRNA and deacylated tRNA ${ }^{\text {Phe }}$ labeled with the nonfluorescent FRET acceptor QSY35 at thio-U8 (tRNA $\left.{ }^{\text {Phe }}(\mathrm{QSY})\right)$. Binding of IF3 ${ }_{166}(\mathrm{Alx} 488)$ to the post-termination complex led to decrease of IF3 fluorescence due to FRET (Fig. 5a, inset). The rate of binding depended on RRF concentration, and the saturating behavior indicated that IF3 binding was limited by a preceding rearrangement. Given the similarity of rates of IF3 binding $\left(0.15 \mathrm{~s}^{-1}\right.$ at saturation with RRF) and ribosome disassembly $\left(0.3 \mathrm{~s}^{-1}\right)^{22}$, the latter step probably limits the rate of IF3 binding.

In contrast, association of IF3 with the post-recycling 30S complex containing mRNA and deacylated tRNA ${ }^{\text {Phe }}(\mathrm{QSY})$ was very rapid and led to a triphasic fluorescence change (Fig. 5b). We obtained rate constants of the individual steps by global fitting of the combined time courses at different concentrations of ribosome complexes, using numerical integration (Fig. 5b). The initial fluorescence decrease was due to quenching of IF $_{166}(\mathrm{Alx} 488)$ fluorescence by the proximity of tRNA $^{\text {Phe }}(\mathrm{QSY})$, reporting on IF3 binding to the ribosome. Association and dissociation rate constants of $670 \mu \mathrm{M}^{-1} \mathrm{~s}^{-1}$ and $4 \mathrm{~s}^{-1}$, respectively (Table 1), were similar to rate constants of IF3 binding to any $30 \mathrm{~S}$ complex. In the second phase, which was relatively slow $\left(0.9 \mathrm{~s}^{-1}\right)$, the fluorescence of IF ${ }_{166}(\mathrm{Alx} 488)$ increased, suggesting that IF3 and tRNA moved apart. The backward rate constant of the second step was very close to zero, suggesting tight binding of IF3 to the $30 \mathrm{~S}$ complex. The final step was slow and reversible (forward and backward rate constants $0.07 \mathrm{~s}^{-1}$ and $0.05 \mathrm{~s}^{-1}$, respectively), similar to rates of tRNA and
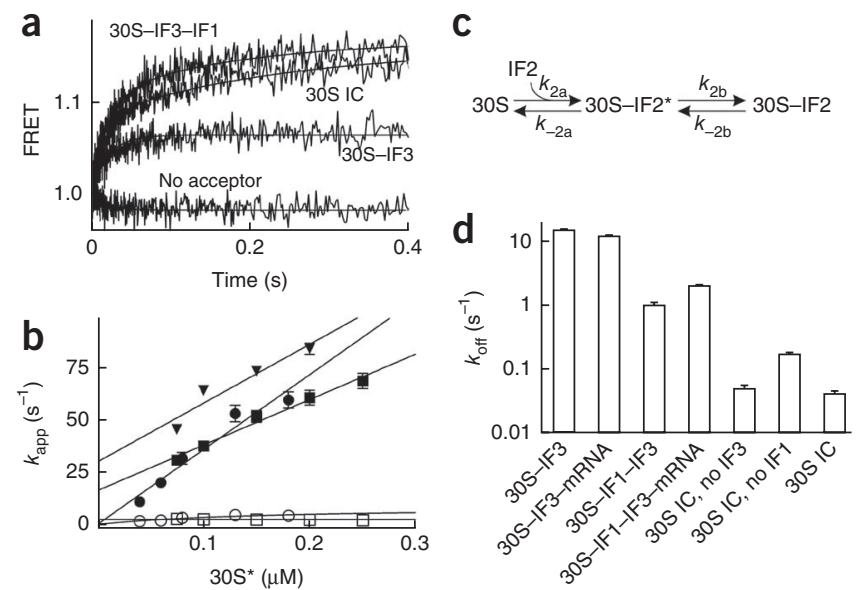
a

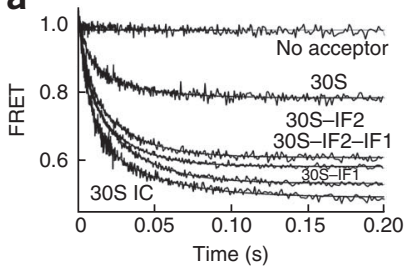

C

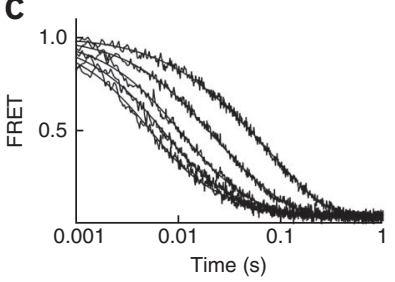

b

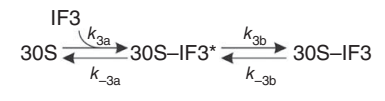

d

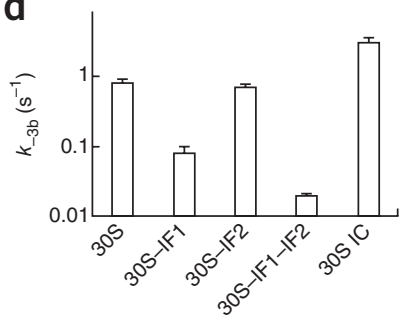

Figure 4 Recruitment of IF3 to the 30S PIC. (a) Time courses of IF3 ${ }_{166}$ (Alx488) binding to the $30 \mathrm{~S}$ subunit as monitored by FRET between IF3 ${ }_{166}$ (Alx488) and mRNA(AttoQ). (b) Two-step scheme of IF3 interaction. (c) Global fitting of time courses of IF3 binding to the 30S-IF1-IF2 complex. The $30 \mathrm{~S}$ subunits (increasing concentration from $0.025 \mu \mathrm{M}$ to $0.2 \mu \mathrm{M}$, right to left) were used to form complexes with IF1 ( $1 \mu \mathrm{M})$, IF2 $(0.3 \mu \mathrm{M})$ with GTP $(0.25 \mathrm{mM})$, or mRNA(AttoQ) $(0.23 \mu \mathrm{M})$ and then rapidly mixed with $\mathrm{IF}_{166}(\mathrm{Alx} 488)(0.01 \mu \mathrm{M})$. Similar qualities of fits were obtained for the other 30 s complexes studied. Smooth lines, global fits of data. (d) Summary of $k_{-3 b}$ values for IF3 determined by chase experiments. Dissociation from $30 \mathrm{~S} \mathrm{IC}$ was measured using FRET from fMet-tRNA ${ }^{\mathrm{fMet}}$ to IF3 and unlabeled 022 mRNA. Values are mean and s.d. from eight to ten time courses. Respective time courses are in Supplementary Figure 2.

mRNA dissociation determined by other methods $\left(0.03-0.07 \mathrm{~s}^{-1}\right)^{22,23}$ and probably reflecting final clearance of the $30 \mathrm{~S}$ subunit. We expected a certain degree of tRNA rebinding, as an excess of tRNA and mRNA was used to form a stable $30 \mathrm{~S}$ post-recycling complex.

\section{mRNA binding to the 30S PIC and 30S IC}

We monitored binding of mRNA to the $30 \mathrm{~S}$ subunit by fluorescence changes of Atto 488 attached at the $3^{\prime}$ end of the mRNA at position +12 downstream from the A of the AUG codon. We tested a collection of mRNA constructs (30 nucleotides long) that differed in the sequence of the ribosome-binding site (Fig. 6a). Mfold analysis ${ }^{24}$ predicted different degrees of secondary structures for the mRNA constructs, with melting temperatures $T_{\mathrm{m}}$ ranging from $25^{\circ} \mathrm{C}$ to $50^{\circ} \mathrm{C}$ (Supplementary Table 3). Through analysis of mRNA mobility by nondenaturing PAGE (Supplementary Fig. 5), we confirmed that the mRNAs varied in secondary structure; their fold was influenced by exchanging only two nucleotides. Binding of mRNA led to fluorescence decrease at a rate largely insensitive to the presence of initiation factors or fMet-tRNA $^{\text {fMet }}$ (Fig. 5b). The concentration dependence of $k_{\text {app }}$ was linear, yielding mRNA association rate constants, $k_{\text {on }}$ (Fig. 6b), in the range of $15-110 \mu \mathrm{M}^{-1} \mathrm{~s}^{-1}\left(20^{\circ} \mathrm{C}\right.$; Fig. $6 \mathbf{c}$, d and Supplementary Table 3). We also conducted some experiments at $37^{\circ} \mathrm{C}$, yielding association rate constants between 40 and $110 \mu \mathrm{M}^{-1} \mathrm{~s}^{-1}$ (Supplementary Table 3), consistent with earlier results ${ }^{10}$. The dissociation rate constants for various mRNAs, obtained without $\mathrm{fMet}_{\mathrm{tRNA}} \mathrm{fMet}^{\mathrm{fm}}$, were in the range of $0.05-3 s^{-1}$ (Fig. 6d and Supplementary Fig. 2d). There was no clear correlation between mRNA sequence features and association or dissociation rates, except for potential secondary structure elements in the m022-derived construct that exhibited slow association and rapid dissociation. This result suggests that the observed variations in association and dissociation rate constants of mRNA-30S PIC complexes without fMet-tRNA ${ }^{\text {fMet }}$ probably

reflect differences in the mRNA secondary structures rather than in the sequences of their ribosome-binding sites.

In the presence of fMet-tRNA ${ }^{\text {fMet }}$, the binding of the mRNAs with cognate start codons was strongly stabilized by codon-anticodon interaction (Fig. 6d), with the exception of m003(AUG), which lacks a Shine-Dalgarno sequence and may be incorrectly positioned (that is, it does not have AUG in the P site). However, as both AUG codon and reading frame are correctly selected on m003 (ref. 11), the mRNA that dissociates faster in the presence of initiator tRNA could be only a subfraction of the total 30S-bound mRNA that does not display the start codon in the P site. If this interpretation is correct, binding of the rest of the mRNA could be strongly stabilized by the fMet-tRNA, as in the case of the Shine-Dalgarno-containing mRNAs, so that its dissociation would become virtually undetectable.

As we expected, binding of fMet-tRNA ${ }^{\text {fMet }}$ was also greatly stabilized by start codon recognition (400-fold for the complex with m002 AUG; Fig. 6e). IF2 and IF1 binding to the 30S IC was also stabilized (70-fold and 10-fold, respectively, for the complex with m002; Fig. 6e). At the same time, the stability of IF3 binding decreased by 10 - to 150-fold, depending on the mRNA (Fig. 4d, 022 mRNA; Fig. 6e, m002), suggesting a global conformational rearrangement of the complex. The exact values of the dissociation rates, and to some extent also the magnitude of the effects, differ depending of the nature of the mRNA ${ }^{7}$.

\section{DISCUSSION}

\section{Kinetic model of 30S PIC assembly}

Using the rapid kinetics approach, we monitored the compositional and temporary dynamics of the 30S PIC assembly in real time (Fig. 7a). The association rate constants, together with the cellular concentrations of the initiation components, determine the order of recruitment of initiation factors, mRNA and fMet-tRNA ${ }^{\text {fMet }}$ to the $30 \mathrm{~S}$ subunit. Using the cellular concentrations of initiation factors $(2-3 \mu \mathrm{M}$ in E. coli ${ }^{25}$; see Online Methods) and the association rate constants summarized in Table 1, we estimated the approximate times at which the factors arrive to the 30 S subunit at $\sim 30 \mathrm{~ms}$ for IF1, $1.3 \mathrm{~ms}$ for IF2 and $<1 \mathrm{~ms}$ for IF3. In comparison, the association rate constant for fMet$\mathrm{tRNA}^{\mathrm{fMet}}$ is $5 \mu \mathrm{M}^{-1} \mathrm{~s}^{-1}$ (ref. 4), and hence its arrival time is $100 \mathrm{~ms}$. The arrival times suggest that IF3 and IF2 are the first to bind to the
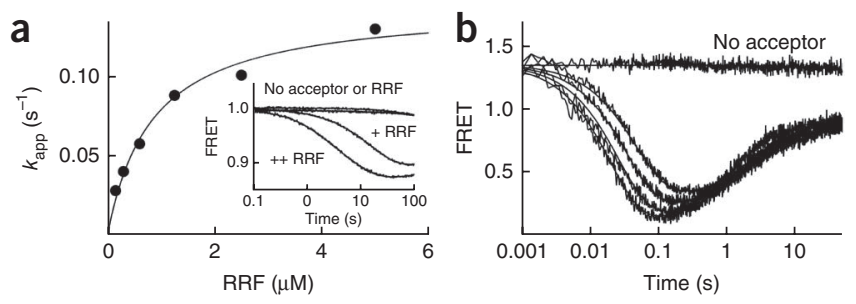

Figure 5 Recruitment of IF3 to ribosome recycling complexes. (a) Dependence of IF3 recruitment to post-termination 70 S complex on RRF concentration. Inset, time courses of reactions at lower (+) and higher $(++)$ concentration of RRF. Post-termination complex prepared from $70 \mathrm{~S}$ ribosomes $(0.1 \mu \mathrm{M})$, poly(U) $\left(0.1 \mathrm{mg} \mathrm{ml}^{-1}\right)$ and tRNAPhe (QSY) $(0.2 \mu \mathrm{M})$ was rapidly mixed at $37{ }^{\circ} \mathrm{C}$ with IF3 ${ }_{166}$ (AIX488) $(0.1 \mu \mathrm{M})$, EF-G $(1.6 \mu \mathrm{M})$ and RRF at indicated concentrations. (b) Time courses of IF3 recruitment to post-recycling $30 \mathrm{~S}$ complexes were obtained by mixing 30 S post-recycling complexes $(0.0125-0.15 \mu \mathrm{M}$, traces from right to left) with $\operatorname{IF}_{166}(\mathrm{Alx} 488)(0.005 \mu \mathrm{M})$ and unlabeled tRNAPhe $^{\text {Ph }}(\mu \mathrm{M})$. Smooth lines, global fits of the time courses. Postrecycling complexes were prepared by incubating $30 \mathrm{~S}$ subunits with poly(U) $\left(0.1 \mathrm{mg} \mathrm{ml}^{-1}\right)$ and tRNAPhe $(\mathrm{QSY})(0.3 \mu \mathrm{M})$. Top curve, control with unlabeled tRNA. 
Figure 6 mRNA recruitment. (a) Sequences of ribosome-binding sites of mRNAs used in this study. Shine-Dalgarno sequences are underlined; initiation codons are bold. (b) Concentration dependence of $k_{\text {app }}$ values of m022 or m003 mRNA binding to $30 \mathrm{~S}$ subunit at $20^{\circ} \mathrm{C}$ and $37^{\circ} \mathrm{C}$. (c) Comparison of association rate constants $\left(k_{\text {on }}\right)$ for binding of various mRNAs to 30 S subunits, 30S-initiation factor complexes (30S IFs) and 30S-initiation factor-fMet-tRNA ${ }^{\mathrm{fMet}}$ complexes (30S IC). Values are mean and s.d. of linear fitting of $k_{\text {app }}$ dependencies. (d) Dissociation rate constants of mRNAs from various $30 \mathrm{~S}$ complexes. mRNAs(Atto488) $(0.05 \mu \mathrm{M})$ were mixed with 30 S subunits $(0.15 \mu \mathrm{M})$ alone, with IF1 $(0.3 \mu \mathrm{M})$, IF2 $(0.3 \mu \mathrm{M})$, GTP $(0.25 \mathrm{mM})$ and IF3 $(0.3 \mu \mathrm{M})(30 \mathrm{~S}-\mathrm{IFs})$, or with initiation factors together with fMet-tRNA ${ }^{\text {fMet }}(0.45 \mu \mathrm{M})(30 \mathrm{SIC})$. Dissociation of the fluorescent mRNA from complexes was initiated by addition of unlabeled mRNA $(1.5 \mu \mathrm{M})$. Asterisk, dissociation rates were too slow to be measured in stopped-flow fluorometer. (e) Dissociation rate constants of initiation factors and fMet-tRNA ${ }^{\mathrm{fMet}}$ as a function of initiation codon. Values in $\mathbf{b}, \mathbf{d}-\mathbf{e}$ are mean and s.d. from eight to ten time courses.

30S subunit, followed by IF1, which binds much more slowly, and finally by fMet-tRNA ${ }^{\text {fMet }}$, which docks to the 30 S subunit-bound IF2 (ref. 4). The kinetically favored assembly pathway probably also pertains to in vivo conditions, as molecular crowding and temperature should affect all association rate constants to a similar extent, and thus the differences in the arrival times of IF3, IF2, IF1 and fMet-tRNA probably persist. Thus, although initiation factors can bind to the 30 S subunit independently of each other, there is a kinetically favored order of assembly events, constituting a preferred route in the multipathway, nonlinear assembly landscape. Similar calculations for mRNAs are difficult, as the concentration of mRNAs varies by orders of magnitude between highly abundant and rare or only temporarily expressed mRNAs ${ }^{26}$ (see below).

The initial recruitment of initiation factors to the $30 \mathrm{~S}$ subunit is followed by conformational adjustments. IF3 undergoes a rearrangement that takes place at a similar rate as the binding of IF1; in principle, the two reactions are independent of one another, although tight docking of IF3 seems to refine the landscape of the 30 S subunit such that IF1 binding is accelerated three times. The rearrangements after the arrival of IF2 may involve conformational adjustments of IF2, domain movements of the $30 \mathrm{~S}$ subunit, unfolding of the mRNA secondary structures and movements of the mRNA from the standby to the initiation site ${ }^{10,13,27}$. Comparison of cryo-EM reconstructions of the 30 S IC obtained with and without IF3 indicated that the CTD of IF2 may be positioned in a different way depending on IF3 (refs. 3,15), suggesting that the IF2 CTD is mobile; this may help in optimizing the alignment of the fMet-tRNA ${ }^{\text {fMet }}$ with respect to the AUG codon and to the incoming 50S subunit.

The arrival time of the mRNA is independent of initiation factors and seems to be characteristic for any given mRNA. The initial a

m022: UUA ACA GGUAUA CAU ACU AUG UUC ACG AUC m002 (AUG): CAC UUA AGG AGG UAU ACU AUG UUC ACG AUC m002 (GGU): CAC UUAAGG AGG UAU ACU GGU UUC ACG AUC m003 (AUG): UUA AUC CCU UCU UAU ACU AUG UUC ACG AUC
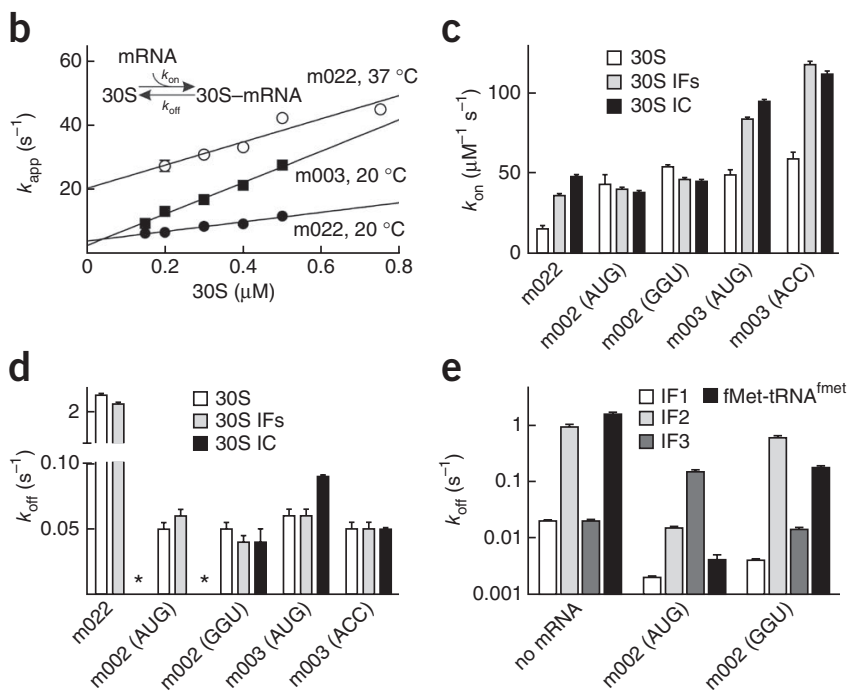

recruitment of mRNA to the 30 S subunit is mainly determined by the concentration and secondary structure at the ribosome-binding site ${ }^{28}$. Our results suggest that, depending on mRNA abundance and accessibility of its ribosome-binding site, mRNAs may join the 30S subunit before, concomitantly with or after the initiation factors.

After the termination of protein synthesis, RRF and EF-G split the $70 \mathrm{~S}$ post-termination complex into $30 \mathrm{~S}-\mathrm{mRNA}-\mathrm{tRNA}$ complex and $50 \mathrm{~S}$ subunits. IF3 binds to the $30 \mathrm{~S}$ complex and promotes dissociation of the deacylated tRNA ${ }^{21,22}$. Our data show that recruitment of IF3 to the ribosome after termination is limited by the rate of ribosome dissociation into subunits. However, after ejection of the $50 \mathrm{~S}$ subunit, IF3 can bind to the 30S-mRNA-tRNA complex with a similar rate as to vacant 30 S subunits. Recruitment of IF3 brings the factor into the vicinity of the tRNA in the $\mathrm{P}$ site. In the following rearrangement step, which precedes the release of tRNA and mRNA from the complex by as much as $15 \mathrm{~s}$, IF3 and the tRNA move apart, forming an intermediate of the $30 \mathrm{~S}$ subunit during recycling that had not been observed before.

\section{Interplay between initiation factors}

The times of IF1-IF3 arrival to the 30S subunit are largely independent of the presence of the other factors or the mRNA. However, both a

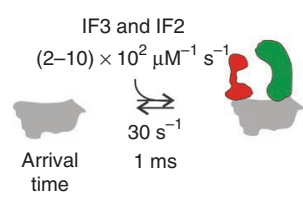

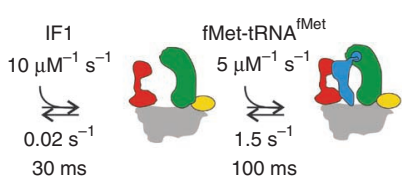

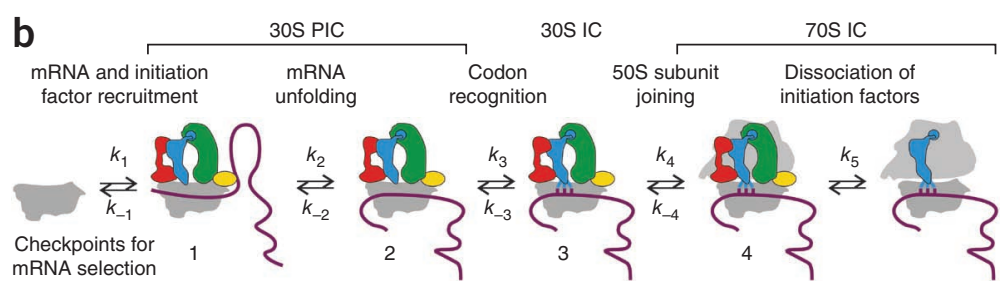

Figure 7 Assembly landscape of 30S PIC formation. (a) Kinetically favored recruitment pathway for initiation factors and fMet-tRNA ${ }^{\mathrm{fMet}}$. IF3 and IF2 are the first to bind to the $30 \mathrm{~S}$ subunit, followed by IF1; values of rate constants are from Table 1 . fMet-tRNA ${ }^{\text {fMet }}$ is recruited to the complete 30S-IFs complex (respective rate constants are from ref. 4). (b) Checkpoints of mRNA selection. The kinetic partitioning checkpoints operate at the stages of mRNA recruitment (checkpoint 1), mRNA unfolding and transition from standby to initiation sites (checkpoint 2), start codon recognition and transition from the 30S PIC to the 30S IC complex (checkpoint 3) and upon 50S subunit joining and transition from the 30 S IC to the 70 S IC (checkpoint 4). Because mRNA recruitment is independent of the IFs and fMet-tRNA ${ }^{\mathrm{fMet}}$, these components are not required to be on the $30 \mathrm{~S}$ PIC before the mRNA binds. 
conformational dynamics and the stability of each factor binding in the 30S PIC are modulated by the respective other two factors. IF3 binding is stabilized four-fold by IF2 and further ten-fold by IF1 (Table 1). As the binding sites of IF1 and IF3 are distant from one another, the effect of IF1 on IF3 is probably due to conformational changes of the $30 \mathrm{~S}$ subunit, for example, movements of the $30 \mathrm{~S}$ subunit head relative to the body, as has been observed in the cryo-EM structure of the complete $30 \mathrm{~S} \mathrm{IC}^{15}$. Interfering with $30 \mathrm{~S}$ subunit motion using the antibiotic streptomycin disrupted communication between the two factors ${ }^{7}$, underscoring the importance of $30 \mathrm{~S}$ dynamics in the joint action of the factors. Structural studies suggest that binding of IF1 may induce a distortion of helix 44 of $16 \mathrm{~S}$ rRNA $^{29}$. Mutations of rRNA residues in that region affect the fidelity of initiation, presumably by impairing communication between IF1 and IF3 (ref. 30). The stabilizing effect of IF1 on IF3 is reciprocal, as the affinity of IF1 binding to the $30 \mathrm{~S}$ subunit is greater with IF3 than without it (this paper and ref. 17).

The dissociation of IF2 from the 30S PIC is ten times slower when IF1 is present in the complex, as compared to the complex without IF1, and, conversely, IF1 binding is stabilized by IF2 (Table 1), consistent with earlier data ${ }^{17}$. The effect may be specific for E. coli IF2, which has an extended NTD that contacts IF1 on the 30 subunit $^{15}$, because the interaction has not been observed with IF2s from thermophilic organisms, which have a much shorter $\mathrm{NTD}^{31}$. The kinetic stabilization results from a rearrangement induced by IF1. The nature of the rearrangement is currently unknown; it may represent the IF1-dependent interaction of the IF2 GTP-binding domain with the 30S PIC, as has been shown by pull-down assays with IF2 truncation mutants ${ }^{32}$, and may be mediated either through direct contact between IF1 and IF2 or by an indirect effect related to the $30 \mathrm{~S}$ subunit conformation. In turn, the conformation of IF2 that stabilizes IF1 binding is induced by IF3 (Table 1), underlining the importance of IF3 for the conformational dynamics of IF2. IF3 and IF2 together bring about a $\geq 35$-fold stabilization of IF1 binding, compared with each of the two factors alone, consistent with cooperative stabilization effects observed earlier ${ }^{17}$.

Taking into account the kinetically favored order of factor binding, an early intermediate of the 30S PIC assembly is a 30S-IF2-IF3 complex, which is formed rapidly and is rather unstable $\left(k_{-23}\right.$ and $k_{-32}$ are $\sim 30 \mathrm{~s}^{-1}$; Fig. 7a). The recruitment of IF1 and the following conformational rearrangement alter the structure of the 30S PIC such that the binding of all factors is stabilized $\left(k_{-123}\right.$ and $k_{-312}$ of $0.02 \mathrm{~s}^{-1}$; $k_{-213}$ of $1 \mathrm{~s}^{-1}$ ). These rearrangements are mainly independent of the mRNA but become crucial for mRNA and start codon selection in the following steps. This notion is supported by mutational analyses that have identified several nucleotides in $16 \mathrm{~S}$ rRNA and residues in IF1 and IF3 that alter the fidelity of translation ${ }^{30,33-35}$. Recruitment

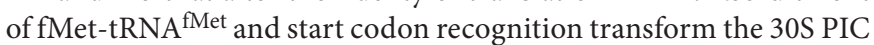
into the mature $30 \mathrm{~S}$ IC, altering the network of interactions in the PIC. Correct start codon selection leads to stabilization of $\mathrm{fMet}-\mathrm{tRNA}{ }^{\mathrm{fMet}}$, mRNA, IF2 and IF1, and to strong destabilization of IF3 (Table 1 and Fig. 6e), in preparation for its dissociation, which occurs during the transition from $30 \mathrm{~S}$ IC to $70 \mathrm{~S} \mathrm{IC}^{7}$. Thus, the affinity switch induced by codon recognition is an important checkpoint that prepares the 30 S IC for 50 S subunit joining.

\section{Checkpoints of mRNA selection at the 30S PIC}

The initiation efficiency, and hence the expression, of individual mRNAs may vary by as much as three orders of magnitude ${ }^{36}$. The efficiency of initial mRNA recruitment to the 30S PIC is proportional to the mRNA concentration and structural features of its ribosomebinding site 28,36 , which account for up to 20 -fold differences in the association rate constants ( $k_{1}$ in Fig. $\mathbf{7 b}$ and ref. 10). As discussed above, the differences are mostly due to secondary structure at the ribosome-binding site, whereas elements such as the strength of the Shine-Dalgarno-anti-Shine-Dalgarno interaction or the presence or absence of the AUG codon are not monitored at this stage (Fig. $\mathbf{7 b}$, checkpoint 1). The recruitment of structured mRNAs may involve docking to the standby site at the platform of the 30 S subunit ${ }^{27,37}$; this allows unfolding of the secondary structure of the mRNA and its adjustment in the mRNA-binding channel. The fate of an mRNA at this point depends on kinetic partitioning between mRNA dissociation ( $k_{-1}$ in Fig. 7 b) and unfolding ( $k_{2}$; checkpoint 2$)$; an mRNA with a strong secondary structure, which unfolds slowly, can be preferentially released from the 30S PIC and exchanged for a different $\mathrm{mRNA}^{10}$. The start codon selection operates the affinity switch that locks the mRNA and fMet-tRNA ${ }^{\text {fMet }}$ on the 30 S subunit and constitutes the next checkpoint for mRNA selection. Here, mRNAs are favored that have a canonical start codon and can be efficiently recognized by fMet-tRNA ${ }^{\mathrm{fMet}}$ (high $k_{3}$ value), whereas mRNAs with noncanonical initiation codons are disfavored (low $k_{3}$ value; checkpoint 3). Finally, the transition from the 30 S IC to the 70 S IC provides the ultimate checkpoint for mRNA selection, in which structural features of the translation initiation region and the presence of cognate mRNA-tRNA pairs are monitored (checkpoint 4$)^{5,7}$. The 30S IC with an optimal mRNA provides a structural landscape that ensures rapid 50S subunit docking ${ }^{3,6,7,15}$ (high $k_{4}$ value), whereas the presence of an unfavorable mRNA delays 70S IC formation, thereby leading to its lower expression ${ }^{7}$. In addition, the 30S PIC, which does not have a proper codon-anticodon interaction, may release the 50S subunit (high $k_{-4}$ value). Thus, the assembly of the 30 S PIC and its transition to the 30S IC and 70S IC provide multiple points at which the fate of an mRNA is decided on the basis of kinetic partitioning between the forward reactions and dissociation of the mRNA from the complex.

\section{METHODS}

Methods and any associated references are available in the online version of the paper.

Note: Supplementary information is available in the online version of the paper.

\section{ACKNOWLEDGMENTS}

We thank W. Wintermeyer for critically reading and shortening the manuscript, C. Kothe for doing some stopped-flow experiments and O. Geintzer, S. Kappler, C. Kothe and T. Wiles for expert technical assistance. We also thank the participants of the methods course on transient kinetics of the Göttingen Graduate School for Neurosciences, Biophysics and Molecular Biosciences for doing some stopped-flow experiments. This work was supported by grants of the Deutsche Forschungsgemeinschaft (M.V.R.) and Italian Ministero dell'Instruzione, dell'Universitá e della Ricerca (C.O.G.).

\section{AUTHOR CONTRIBUTIONS}

P.M., C.O.G. and M.V.R. conceived the research. P.M. and M.V.R. designed the experiments and analyzed the data. P.M., C.M. and L.F. prepared materials and conducted experiments. P.M., C.O.G. and M.V.R. wrote the paper.

\section{COMPETING FINANCIAL INTERESTS}

The authors declare no competing financial interests.

Published online at http://www.nature.com/doifinder/10.1038/nsmb.2285 Reprints and permissions information is available online at http://www.nature.com/ reprints/index.html.

1. Gualerzi, C.O. et al. Initiation factors in the early events of mRNA translation in bacteria. Cold Spring Harb. Symp. Quant. Biol. 66, 363-376 (2001).

2. Laursen, B.S., Sorensen, H.P., Mortensen, K.K. \& Sperling-Petersen, H.U. Initiation of protein synthesis in bacteria. Microbiol. Mol. Biol. Rev. 69, 101-123 (2005).

3. Simonetti, A. et al. A structural view of translation initiation in bacteria. Cell. Mol. Life Sci. 66, 423-436 (2009). 
4. Milón, P. et al. The ribosome-bound initiation factor 2 recruits initiator tRNA to the 30 S initiation complex. EMBO Rep. 11, 312-316 (2010).

5. Antoun, A., Pavlov, M.Y., Lovmar, M. \& Ehrenberg, M. How initiation factors tune the rate of initiation of protein synthesis in bacteria. EMBO J. 25, 2539-2550 (2006).

6. Grigoriadou, C., Marzi, S., Kirillov, S., Gualerzi, C.O. \& Cooperman, B.S. A quantitative kinetic scheme for $70 \mathrm{~S}$ translation initiation complex formation. J. Mol. Biol. 373, 562-572 (2007).

7. Milón, P., Konevega, A.L., Gualerzi, C.O. \& Rodnina, M.V. Kinetic checkpoint at a late step in translation initiation. Mol. Cell 30, 712-720 (2008).

8. Marshall, R.A., Aitken, C.E. \& Puglisi, J.D. GTP hydrolysis by IF2 guides progression of the ribosome into elongation. Mol. Cell 35, 37-47 (2009).

9. Tomsic, J. et al. Late events of translation initiation in bacteria: a kinetic analysis. EMBO J. 19, 2127-2136 (2000)

10. Studer, S.M. \& Joseph, S. Unfolding of mRNA secondary structure by the bacterial translation initiation complex. Mol. Cell 22, 105-115 (2006).

11. Calogero, R.A., Pon, C.L., Canonaco, M.A. \& Gualerzi, C.O. Selection of the mRNA translation initiation region by Escherichia coli ribosomes. Proc. Natl. Acad. Sci. USA 85, 6427-6431 (1988).

12. Grigoriadou, C., Marzi, S., Pan, D., Gualerzi, C.O. \& Cooperman, B.S. The translational fidelity function of IF3 during transition from the $30 \mathrm{~S}$ initiation complex to the $70 \mathrm{~S}$ initiation complex. J. Mol. Biol. 373, 551-561 (2007).

13. La Teana, A., Gualerzi, C.O. \& Brimacombe, R. From stand-by to decoding site. Adjustment of the mRNA on the $30 \mathrm{~S}$ ribosomal subunit under the influence of the initiation factors. RNA 1, 772-782 (1995).

14. Milón, P. et al. Transient kinetics, fluorescence, and FRET in studies of initiation of translation in bacteria. Methods Enzymol. 430, 1-30 (2007).

15. Julián, P. et al. The cryo-EM structure of a complete $30 \mathrm{~S}$ translation initiation complex from Escherichia coli. PLoS Biol. 9, e1001095 (2011).

16. Celano, B., Pawlik, R.T. \& Gualerzi, C.O. Interaction of Escherichia coli translationinitiation factor IF-1 with ribosomes. Eur. J. Biochem. 178, 351-355 (1988).

17. Zucker, F.H. \& Hershey, J.W. Binding of Escherichia coli protein synthesis initiation factor IF1 to 30 S ribosomal subunits measured by fluorescence polarization. Biochemistry 25, 3682-3690 (1986).

18. Misselwitz, R. et al. The fMet-tRNA binding domain of translational initiation factor IF2: role and environment of its two Cys residues. FEBS Lett. 459, 332-336 (1999).

19. Weiel, J. \& Hershey, J.W. The binding of fluorescein-labeled protein synthesis initiation factor 2 to Escherichia coli $30 \mathrm{~S}$ ribosomal subunits determined by fluorescence polarization. J. Biol. Chem. 257, 1215-1220 (1982).

20. Petrelli, D. et al. Translation initiation factor IF3: two domains, five functions, one mechanism? EMBO J. 20, 4560-4569 (2001).
21. Karimi, R., Pavlov, M.Y., Buckingham, R.H. \& Ehrenberg, M. Novel roles for classical factors at the interface between translation termination and initiation. Mol. Cell $\mathbf{3}$ 601-609 (1999).

22. Peske, F., Rodnina, M.V. \& Wintermeyer, W. Sequence of steps in ribosome recycling as defined by kinetic analysis. Mol. Cell 18, 403-412 (2005).

23. Antoun, A., Pavlov, M.Y., Lovmar, M. \& Ehrenberg, M. How initiation factors maximize the accuracy of tRNA selection in initiation of bacterial protein synthesis. Mol. Cell 23, 183-193 (2006).

24. Zuker, M. Mfold web server for nucleic acid folding and hybridization prediction. Nucleic Acids Res. 31, 3406-3415 (2003).

25. Bremer, H. \& Dennis, P.P. Modulation of chemical composition and other parameters of the cell by growth rate. in Escherichia coli and Salmonella typhimurium. Cellular and Molecular Biology (ed. Neidherdt, F.C.) 1527-1542 (American Society for Microbiology, Washington, DC, 1987).

26. Passalacqua, K.D. et al. Structure and complexity of a bacterial transcriptome. J. Bacteriol. 191, 3203-3211 (2009).

27. Yusupova, G., Jenner, L., Rees, B., Moras, D. \& Yusupov, M. Structural basis for messenger RNA movement on the ribosome. Nature 444, 391-394 (2006).

28. Kudla, G., Murray, A.W., Tollervey, D. \& Plotkin, J.B. Coding-sequence determinants of gene expression in Escherichia coli. Science 324, 255-258 (2009).

29. Carter, A.P. et al. Crystal structure of an initiation factor bound to the 30 S ribosomal subunit. Science 291, 498-501 (2001).

30. Qin, D. \& Fredrick, K. Control of translation initiation involves a factor-induced rearrangement of helix 44 of 16 S ribosomal RNA. Mol. Microbiol. 71, 1239-1249 (2009).

31. Kapralou, S. et al. Translation initiation factor IF1 of Bacillus stearothermophilus and Thermus thermophilus substitute for Escherichia coli IF1 in vivo and in vitro without a direct IF1-IF2 interaction. Mol. Microbiol. 70, 1368-1377 (2008).

32. Caserta, E. et al. Translation initiation factor IF2 interacts with the $30 \mathrm{~S}$ ribosomal subunit via two separate binding sites. J. Mol. Biol. 362, 787-799 (2006).

33. Belotserkovsky, J.M., Dabbs, E.R. \& Isaksson, L.A. Mutations in 16S rRNA that suppress cold-sensitive initiation factor 1 affect ribosomal subunit association. FEBS J. 278, 3508-3517 (2011).

34. Qin, D., Abdi, N.M. \& Fredrick, K. Characterization of 16S rRNA mutations that decrease the fidelity of translation initiation. RNA 13, 2348-2355 (2007).

35. Surkov, S., Nilsson, H., Rasmussen, L.C., Sperling-Petersen, H.U. \& Isaksson, L.A. Translation initiation region dependency of translation initiation in Escherichia coli by IF1 and kasugamycin. FEBS J. 277, 2428-2439 (2010).

36. McCarthy, J.E. \& Gualerzi, C. Translational control of prokaryotic gene expression. Trends Genet. 6, 78-85 (1990).

37. Marzi, S. et al. Structured mRNAs regulate translation initiation by binding to the platform of the ribosome. Cell 130, 1019-1031 (2007). 


\section{ONLINE METHODS}

Biochemical methods. All measurements were made in buffer A (50 mM Tris$\mathrm{HCl}, \mathrm{pH} 7.5,70 \mathrm{mM} \mathrm{NH}_{4} \mathrm{Cl}, 30 \mathrm{mM} \mathrm{KCl}$ and $7 \mathrm{mM} \mathrm{MgCl}_{2}$ ) at $20^{\circ} \mathrm{C}$ unless stated otherwise. Ribosomes, proteins and tRNA were from E. coli. Ribosomal subunits were prepared from purified $70 \mathrm{~S}$ ribosomes ${ }^{14,38}$ by sucrose gradient centrifugation in a zonal rotor (Ti 15, Beckman); the activity of reassociated $30 \mathrm{~S}$ and $50 \mathrm{~S}$ subunits was $>95 \%$ in $\mathrm{fMet}^{-\mathrm{tRNA}} \mathrm{fMet}^{\mathrm{f}}$ binding and peptide bond formation. $30 \mathrm{~S}$ subunits were reactivated in buffer A with $20 \mathrm{mM} \mathrm{MgCl}_{2}$ for $1 \mathrm{~h}$ at $37^{\circ} \mathrm{C}$. fMettRNA $^{\text {fMet }}$ was purified by HPLC and was $95 \%$ aminoacylated and formylated ${ }^{14}$. Unless stated otherwise, we used a 124 -nucleotide 022 mRNA $^{11,14}$ that was produced by T7 RNA-polymerase transcription. Short fluorescent mRNAs were purchased from Iba. Preparation of single-cysteine mutants of IF1 and IF3 and fluorescence labeling of all components was carried out as described ${ }^{14}$. The alpha isoform of IF2 was used. The activity of the labeled components was verified by biochemical tests ${ }^{7,14}$ (see also Supplementary Fig. 1). RRF and EF-G were prepared as described ${ }^{22}$. The post-termination complex was prepared by binding of $70 \mathrm{~S}$ ribosomes to poly(U) with tRNA ${ }^{\mathrm{Phe}}(\mathrm{QSY} 35)$ in the $\mathrm{P}$ site in buffer A with $20 \mathrm{mM} \mathrm{MgCl}_{2}$ for $1 \mathrm{~h}$ at $37^{\circ} \mathrm{C}$; the final $\mathrm{MgCl}_{2}$ concentration in the stopped-flow experiments was adjusted to $7 \mathrm{mM}$. The $30 \mathrm{~S}$ post-recycling complex was prepared in the same way except for tRNA concentration ( $0.3 \mu \mathrm{M}$; see legend to Fig. 5).

Kinetic experiments. Fluorescence stopped-flow measurements were done using a SX-20MV stopped-flow apparatus (Applied Photophysics). Experiments were carried out by rapidly mixing equal volumes ( $60 \mu \mathrm{l}$ each) of reactants in buffer $\mathrm{A}$ at $20^{\circ} \mathrm{C}$. In a single experiment, 1,000-4,000 data points were acquired in logarithmic sampling mode. Excitation wavelength was $465 \mathrm{~nm}$. Fluorescence emission and FRET were measured after using 500-nm or 590-nm cutoff filters to measure fluorescence of donor or acceptor, respectively. Time courses of binding to $30 \mathrm{~S}$ complexes with various combinations of initiation ligands were obtained for each labeled factor and mRNA. Apparent rate constants $\left(k_{\text {app }}\right)$ were determined under pseudo-first-order conditions at increasing concentrations of either the labeled factor (for example, IF1) or the respective ribosome complex. The concentration of complex or factor that carried the FRET donor was kept constant, whereas the concentration of the FRET acceptor varied. Data were evaluated by fitting the exponential function $F=F_{\infty}+A \exp \left(-k_{\text {app }} t\right)$, with a characteristic time constant $k_{\text {app }}$, the amplitude of the signal change $A$, the final signal $F_{\infty}$ and the fluorescence at time $t, F$. If necessary, additional exponential terms were included. Calculations were done using TableCurve software (Jandel Scientific) or Prism (Graphpad Software). All values are mean \pm s.d. from eight to ten time courses. For a simple one-step reversible binding mechanism, association and dissociation rate constants were estimated by linear fitting of the concentration dependence of the $k_{\text {app }}$ values. In cases in which more than one exponential term was required to fit the time courses, we additionally estimated the values of rate constants by global fitting of time courses measured at different concentration of complexes by numerical integration using Scientist for Windows (MicroMath Scientific Software).

Dissociation rate constants were also determined by chase experiments in which we mixed preformed $30 \mathrm{~S}$ complexes containing fluorescent components with an excess of an unlabeled component in the stopped-flow apparatus. Where necessary, that is, for two-step association reactions, the measured dissociation values were corrected for the extent the respective complex was populated using rate constants from numerical integration (Table 1). Whenever more than one FRET pair was used to measure the kinetics of interactions, very similar values of association and dissociation rate constants were obtained. The arrival times $\tau$ were calculated from the bimolecular association rate constant and the concentration of the respective factor using the following concentrations: IF1, $2.5 \mu \mathrm{M}$; IF2, $3 \mu \mathrm{M}$; IF3, $2 \mu \mathrm{M}$; and fMet-tRNA ${ }^{\mathrm{fMet}}, 2 \mu \mathrm{M}$ (ref. 25).

To calculate rate constants by global fitting, time courses obtained at several different concentrations of ligands (as indicated in respective figure legends) were combined with time courses of dissociation obtained by chase. The data were fitted by numerical integration using Scientist for Windows software. For IF3 binding to the 30S, 30S-IF1, 30S-IF2, 30S-IF1-IF2 and 30S IC, the kinetic scheme of Figure $3 \mathbf{b}$ was used, with variables for the rate constants $k_{3 \mathrm{a}}, k_{-3 \mathrm{a}}, k_{3 \mathrm{~b}}$ and $k_{-3 \mathrm{~b}}$ and fluorescence factors $\mathrm{Fa}$ (fluorescence of free IF3), $\mathrm{Fc}$ (IF3 bound in the 30 S-IF3* state, Fig. 4 b) and Fd (IF3 bound to the 30S-IF3 complex, Fig. 4b). To normalize the binding data sets, fluorescence was set to 1 at time 0 and to 0 at infinite time for each time course of the titration experiment (Fig. 4c), and all time courses were fitted with a common set of rate constants and fluorescence factors. An additional set of fluorescence factors was used for the chase data set. The values of the rate constants determined analytically (Supplementary Fig. 4) were used as initial values for fitting. The fitting yields a unique solution for rate constants and for fluorescence factors. Identical rate constants were obtained if arbitrary values were used as initial guesses for rate constants or fluorescence factors (for example, when all values were set to 1); however, in this case the calculations took much longer. The variations in fluorescence factors did not affect the solution for values of rate constants, as the same results were obtained when raw stopped-flow data were used without rescaling; however, in this case a separate set of fluorescence factors had to be assumed for each time course.

The time courses of IF3 binding to the post-termination complex (Fig. 5b) were fitted to a three-step scheme, $A+B \leftrightarrow C \leftrightarrow D \leftrightarrow E$, with a set of six rate constants and four fluorescence factors $F \mathrm{a}, \mathrm{Fc}, \mathrm{Fd}$ and $\mathrm{Fe}$. The time courses were normalized to start at the same value at time 0 and end at the same value (set to 1 ) at infinite time. The initial guesses for the rate constants were arbitrary; identical solutions were obtained starting from various initial guesses.

38. Rodnina, M.V. \& Wintermeyer, W. GTP consumption of elongation factor Tu during translation of heteropolymeric mRNAs. Proc. Natl. Acad. Sci. USA 92, 1945-1949 (1995). 